\title{
Validation study of automated oscillometric measurement of the ankle-brachial index for lower arterial occlusive disease by comparison with computed tomography angiography
}

\begin{abstract}
Shigeo Ichihashi ${ }^{1}$, Tomoko Hashimoto ${ }^{2}$, Shinichi Iwakoshi ${ }^{1}$ and Kimihiko Kichikawa ${ }^{1}$
The ankle-brachial index ( $\mathrm{ABI}$ ) determined by the oscillometric method has been shown to reliably detect peripheral arterial disease (PAD), with highly correlations with the Doppler method. However, most of these studies were shown in cohorts with a small number of PAD patients, and no imaging studies have been performed. The purpose of this study is to evaluate the diagnostic accuracy and optimal threshold of oscillometric ABI for detecting PAD using computed tomography angiography (CTA) as a gold standard in a cohort that consists mostly of PAD patients. This retrospective study included 108 consecutive patients with 216 limbs. ABI measured by an oscillometric device was compared with CTA. The diagnostic accuracy of oscillometric $A B I$ to detect $\geqslant 50 \%$ and $\geqslant 75 \%$ stenosis confirmed by CTA and the optimal ABI cutoff values were evaluated using receiver operating characteristic (ROC) curve analysis. The oscillometric $A B I$ could not be measured in nine limbs. The mean $A B I$ was $0.72 \pm 0.31$. The areas under the ROC curves (AUCs) for detecting $\geqslant 50 \%$ and $\geqslant 75 \%$ stenosis with oscillometric $\mathrm{ABI}$ were 0.919 and 0.918 , respectively. The optimal $\mathrm{ABI}$ cutoff values to detect these levels of stenosis were 0.99 (sensitivity, $90 \%$ : specificity, $85 \%$ ) and 0.87 (sensitivity, $84 \%$ : specificity, $89 \%$ ), respectively. If patients with diabetes mellitus (DM) were analyzed separately, the AUC for detecting $\geqslant 75 \%$ stenosis was 0.888 . Oscillometric ABI had a high diagnostic accuracy to detect PAD using CTA as a gold standard. The diagnostic ability of ABI to detect PAD could be impaired by the presence of DM.
\end{abstract}

Hypertension Research (2014) 37, 591-594; doi:10.1038/hr.2014.34; published online 6 March 2014

Keywords: CTA; oscillometric ABI; peripheral arterial disease

\section{INTRODUCTION}

The ankle-brachial index (ABI) is a simple and noninvasive measure to assess the patency of the arteries of the lower extremity and can predict cardiovascular morbidity and mortality. ${ }^{1,2}$ ABI obtained by Doppler ultrasonography is considered the gold standard; however, this approach is time consuming, requires trained observers and has an intra-observer variability of $10 \%$, which precludes its routine use in general practice. ${ }^{3}$

Alternatively, automated oscillometric blood pressure devices have been developed for ABI measurement. This method reduces the measurement time, requires little observer training ${ }^{4}$ and significantly increases the reproducibility of $\mathrm{ABI}$ estimates compared with the manual approach. ${ }^{5}$ Several studies including a recent meta-analysis have evaluated the performance of the oscillometric method using the Doppler method as a reference standard. These studies demonstrated that the oscillometric method was accurate, as there was a high correlation between oscillometric and Doppler methods. ${ }^{4-6}$ However, most of the subjects included in those studies were healthy individuals and only a small fraction had peripheral arterial disease (PAD). Furthermore, no imaging studies were carried out to define the severity of lesions based on anatomy. A recent statement from American Heart Association (AHA) $)^{2}$ questioned the validity of the oscillometric $\mathrm{ABI}$, especially in $\mathrm{PAD}$ patients in whom the oscillometric method tended to overestimate the actual ankle pressure and could not detect low pressure $(<50 \mathrm{~mm} \mathrm{Hg}){ }^{7}$ Furthermore, the diagnostic performance of oscillometric ABI tended to decrease in patients with comorbidities such as diabetes mellitus $(\mathrm{DM})^{8}$ or renal failure that required hemodialysis. ${ }^{9}$ Thus, a validation study of oscillometric $\mathrm{ABI}$ is mandatory in a cohort that includes a large number of PAD patients. The purpose of this study is to evaluate the diagnostic accuracy and optimal threshold of oscillometric ABI for detecting arterial occlusive disease using computed tomography angiography (CTA) as a gold standard in a cohort that consists mostly of PAD patients.

${ }^{1}$ Department of Radiology, Nara Medical University, Nara, Japan and 2Product Development Strategy HQ Technology Development Department, Omron Healthcare Co, Ltd., Kyoto, Japan

Correspondence: Dr S Ichihashi, Department of Radiology, Nara Medical University, 840 Shijo-cho, Kashihara, Nara 634-8521, Japan.

E-mail: shigeoichihashi@yahoo.co.jp

Received 6 September 2013; revised 8 December 2013; accepted 16 December 2013; published online 6 March 2014 


\section{METHODS}

\section{Study population}

This retrospective study included 108 patients with 216 limbs. The patients were referred to our department based on a suspicion of PAD from June 2010 to December 2012. Patients who had a history of vascular surgery or endovascular treatment for PAD were excluded. Patient demographics are shown in Table 1. Informed consent was obtained from all participants. The institutional review board in our university approved this study.

\section{ABI Measurements}

The ABI measurements were obtained after the patients had rested for $15 \mathrm{~min}$ in the supine position in a room with a comfortable temperature $\left(19-20^{\circ} \mathrm{C}\right)$ and refrained from smoking, heavy exercise and drinking alcohol or caffeinated beverages for at least $2 \mathrm{~h}$ before the examination.

ABI was measured in all patients using an automated oscillometric device (form-III PWV/ABI, Omron Healthcare, Kyoto, Japan). The oscillometric cuffs were appropriately sized for arms and ankles. Systolic blood pressure was measured with the cuff placed on one arm and above the ankles in both legs using the modified oscillometric pressure-sensor method. Ankle pressures were measured over the dorsalis and posterior tibial arteries. The highest ankle pressure was used for calculating the ABI. All measurements using the oscillometric method were performed by the same investigator with 10 years of experience in $\mathrm{ABI}$ measurements.

\section{CT angiography}

All patients underwent CTA within 1 month before or after the ABI measurement. All examinations were performed with a dual-source 64-slice CT scanner (Somatom Definition; Siemens Medical Solutions, Erlangen, Germany). The CTA protocol consisted of plain, arterial and venous phases encompassing the abdomen and lower extremity. A total of $100-150 \mathrm{ml}$ of nonionic-iodinated contrast medium was administered at a flow rate of $4 \mathrm{ml} \mathrm{s}^{-1}$. Arterial phase scanning was performed using a bolus-tracking technique. The arterial phase data acquisition was initiated $6 \mathrm{~s}$ after the attenuation reached a predefined threshold of 120 Housenfield units. Delayed phase dual-energy CT scanning was performed with a standard delay of $70 \mathrm{~s}$ after the beginning of the contrast injection.

Analysis of the contrast-enhanced CT images was performed by a single radiologist with 10 years of experience in vascular imaging who was blinded to the patient's clinical symptom or ABI value. Axial images and maximum intensity projection (MIP) images were used for the evaluation of the stenosis. Stenosis of arteries were graded with a four-point scale (grade 1, 0-50\%; grade 2, 50-75\%; grade 3, 75-99\%; grade 4, 100\%) in each of the following arterial segments: aortoiliac, femoropopliteal and below the knee. Grading was performed by visual assessment, which has been commonly used in the past literatures. ${ }^{10,11}$ There are commonly three main arteries below the knee (BTK arteries): anterior tibial, posterior tibial and peroneal. We used the

\section{Table 1 Patient characteristics}

\begin{tabular}{lc}
\hline Age (years) & $71.2 \pm 8.1$ \\
Female gender & $14(13 \%)$ \\
Symptom ( $\mathrm{n}=207$ limbs) & \\
No symptom & $45(22 \%)$ \\
Intermittent claudication & $153(74 \%)$ \\
Critical limb ischemia & $9(4 \%)$ \\
& \\
Comorbidities ( $\mathrm{n}=108$ patients) & \\
HT & $95 \%$ \\
DM & $54 \%$ \\
CAD & $39 \%$ \\
CVD & $26 \%$ \\
HD & $16 \%$ \\
AF & $10 \%$ \\
\hline Abbreviations: AF, atrial fibrillation; CAD, cardiovascular disease; CVD, cerebrovascular disease; \\
DM, diabetes mellitus; HD, hemodialysis; HT, hypertension.
\end{tabular}

Abbreviations: AF, atrial fibrillation; CAD, cardiovascular dis
DM, diabetes mellitus; HD, hemodialysis; HT, hypertension. artery with the least stenosis to grade all three BTK arteries together, according to the one straight line flow concept.

\section{Definition}

Hypertension was defined as systolic blood pressure $\geqslant 140 \mathrm{~mm} \mathrm{Hg}$ and/or diastolic blood pressure $\geqslant 90 \mathrm{~mm} \mathrm{Hg}$ or ongoing medication therapy. DM was defined as HbAlc $>6.5 \%$, random plasma glucose $>200 \mathrm{mg} \mathrm{dl}^{-1}$ or current treatment with oral hypoglycemic drugs or insulin injection. Coronary artery disease was defined as stable angina with documented coronary artery disease, history of percutaneous coronary intervention, history of coronary artery bypass graft surgery or previous myocardial infarction. Cerebrovascular disease was defined as a history of transient ischemic attack or stroke. Atrial fibrillation was diagnosed with an echocardiogram, which demonstrates the absence of $\mathrm{P}$ wave together with an irregular ventricular rate.

\section{Parameters investigated}

The following parameters were investigated: (1) diagnostic accuracy (sensitivity and specificity) of oscillometric $\mathrm{ABI}$ to detect $\geqslant 50 \%$ and $\geqslant 75 \%$ stenosis demonstrated in any lower extremity artery from the aortic bifurcation to the tibial arteries using CTA as a gold standard and (2) correlation of the ABI value and the severity of stenosis. The diagnostic accuracy was also evaluated with DM patients separately, in whom diagnostic accuracy of oscillometric ABI was reported to be lower than nonDM patients. ${ }^{8}$

\section{Statistical analysis}

Data are presented as the mean \pm s.d. The diagnostic accuracy of oscillometric ABI was evaluated using receiver operating characteristic (ROC) curves. All statistical tests were performed using SPSS version 20 software (IBM, New York, NY, USA)

\section{RESULTS}

The oscillometric ABI value could not be measured in 9 out of 216 limbs: eight limbs due to diffuse calcification of the arterial wall and three limbs due to low blood pressure (two limbs had both of these conditions). These nine limbs were excluded from the analysis. There were 162 of 207 limbs (78\%) that had at least one lesion with $>50 \%$ stenosis. The mean ABI and mean systolic pressure of the brachial artery were $0.72 \pm 0.31 \mathrm{~mm} \mathrm{Hg}$ and $140 \pm 18 \mathrm{~mm} \mathrm{Hg}$, respectively. Detailed ABI values according to the symptoms, stenosis grade are shown in Table 2.

\section{Diagnostic accuracy (sensitivity and specificity) of oscillometric}

ABI.

When CTA was used as the gold standard to define $\geqslant 50 \%$ and $\geqslant 75 \%$ stenosis, the respective areas under the ROC curves (AUCs)

Table 2 Mean ABI values according to the symptoms and degree of stenosis

\begin{tabular}{lcc} 
Symptom & Number of limbs (\%) & Median (minimum, maximum) \\
\hline No symptom & $45(22 \%)$ & $1.03 \pm 0.18$ \\
Intermittent claudication & $153(74 \%)$ & $0.74 \pm 0.22$ \\
Critical limb ischemia & $9(4 \%)$ & $0.98 \pm 0.54$ \\
Total & 207 & $0.72 \pm 0.31$ \\
& & \\
Degree of stenosis & & $1.11 \pm 0.19$ \\
Grade 1 & $45(22 \%)$ & $0.97 \pm 0.20$ \\
Grade 2 & $28(13 \%)$ & $0.74 \pm 0.19$ \\
Grade 3 & $70(34 \%)$ & $0.63 \pm 0.17$ \\
Grade 4 & $64(31 \%)$ &
\end{tabular}

Abbreviation: $A B I$, ankle-brachial index.

(Grade 1, 0-50\%; Grade 2, 50-75\%; Grade 3, 75-99\%; Grade 4, 100\%). 
were 0.919 (95\% confidence interval (CI): $0.880-0.959)$ and 0.918 (95\% CI: 0.879-0.957) (Figure 1). A cutoff value of 0.99 was found to be optimal for detecting $\geqslant 50 \%$ stenosis (sensitivity, $90 \%$; specificity, $85 \%$ ), whereas a cutoff value of 0.87 was optimal for detecting $\geqslant 75 \%$ stenosis (sensitivity, $84 \%$; specificity, $89 \%$ ). If only patients with DM were analyzed separately, AUC for detecting $\geqslant 75 \%$ stenosis was 0.888 (95\% CI: $0.825-0.952)$ with an optimal cutoff value of 0.86 (sensitivity, $81 \%$; specificity, $84 \%$ ).

\section{$A B I$ value depending on the severity of stenosis.}

With a greater stenosis of the artery in the lower extremity, the ABI value tended to become lower (Figure 2, Table 2).

\section{DISCUSSION}

Oscillometric ABI can be easily performed by clinical assistants and reduce the examination time, as compared with the Doppler method. Many investigators ${ }^{4-6,12-14}$ have found that the oscillometric method is a useful tool for clinical assessment of PAD that can replace Doppler measurements in most clinical situations. However, other studies have found that oscillometry overestimates ankle pressure and is unreliable. ${ }^{15,16}$ In those reports, a major problem was that the oscillometric method tended to give higher values than the Doppler method as it could not detect low ankle pressure $(<50 \mathrm{~mm} \mathrm{Hg})$ compared with the Doppler method. ${ }^{2,5,14}$ However, most of the studies only determined the correlation between Doppler and oscillometric $\mathrm{ABI}$, without direct comparison with anatomical information. In the present study, a favorable diagnostic accuracy of oscillometric ABI for detecting $\geqslant 50 \%$ and $\geqslant 75 \%$ stenosis was demonstrated when CTA was used as a gold standard. The value of oscillometric ABI has been reported to be higher than Doppler ABI at low ankle pressure; however, the present results demonstrated the reliability of the oscillometric method compared with CTA in a cohort with a high prevalence of PAD. Guo et al. ${ }^{17}$ compared oscillometric ABI with digital subtraction angiography and concluded that $\mathrm{ABI}$ was an accurate and reliable noninvasive alternative to conventional digital subtraction angiography for assessing lower extremity arteries. However, only $7 \%$ of the patients had PAD in that study, whereas 65\% (134/207), 78\% (162/207) of the limbs had $\geqslant 75 \%, \geqslant 50 \%$ stenosis, respectively, in our study.

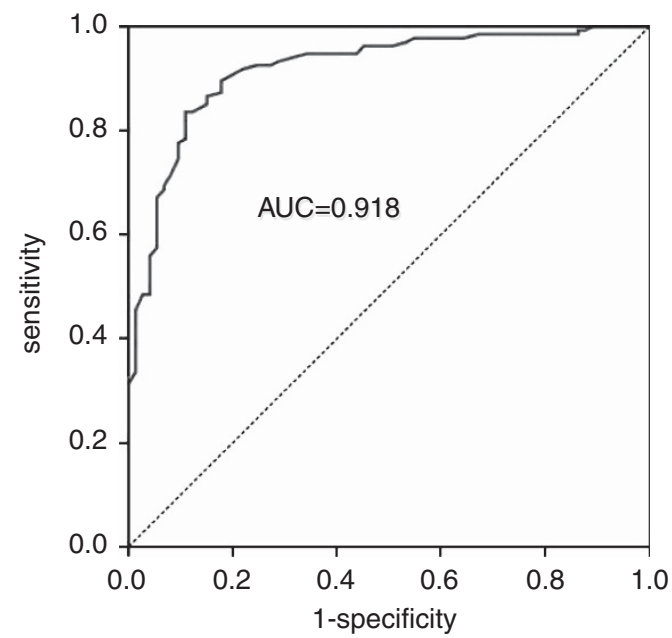

Figure 1 ROC curve of the ABI for diagnosing PAD when CTA was used as the gold standard for defining $\geqslant 75 \%$ stenosis. A full color version of this figure is available at the Hypertension Research journal online.
An ABI cutoff value of 0.99 was found to be optimal for detecting $\geqslant 50 \%$ stenosis (sensitivity, 90\%; specificity, $85 \%$ ), whereas a cutoff value of 0.87 was optimal for detecting $\geqslant 75 \%$ stenosis (sensitivity, $84 \%$; specificity, $89 \%$ ). Guo et al. ${ }^{17}$ reported that 0.95 was the optimal cutoff value for detecting $50 \%$ stenosis. Clairotte et al. ${ }^{8}$ found that the optimal cutoff value for detecting 50\% stenosis was 1.02 . Our optimal cutoff values are consistent with these previous studies. The recent statement from the $\mathrm{AHA}^{2}$ recommended that subjects with an $\mathrm{ABI}$ between 0.91 and 1.00 should be considered 'borderline' because the risk of cardiovascular mortality might be slightly higher than normal. In total, 50\% stenosis doesn't necessarily impede the arterial flow, but it means atherosclerotic burden in the artery. From the point of cardiovascular event prevention, our optimal ABI cutoff of 0.99 for the detection of $\geqslant 50 \%$ stenosis supports the AHA recommendation, with 1.00 as a threshold between normal and borderline.

When we separately analyzed patients with DM, the AUC for detecting $\geqslant 75 \%$ was 0.888 , inferior to that of the entire patient cohort (AUC, 0.918). Clairotte et al. ${ }^{8}$ reported a lower diagnostic performance with a higher cutoff value of oscillometric ABI in diabetic patients. The reason for decreased diagnostic accuracy might be related to poor arterial compressibility resulting from stiffness and calcification, which may occur commonly in DM. ${ }^{18,19}$ In the present study, eight of nine patients in whom ABI values could not be correctly measured had diffuse arterial calcification.

An interesting finding in the present study was that the optimal ABI cutoff value showed a tendency to decrease with increasing severity of stenosis in patients with PAD. Guo et al. ${ }^{17}$ also reported this finding. However, it is difficult to predict the degree of stenosis based only on the ABI value, because not only the degree of stenosis but also the number of stenotic segments or amount of calcification in the arterial wall may affect the ABI value. Further study is required to resolve these issues.

This study has several potential limitations. All data were analyzed retrospectively at a single facility, which limits the power of the study. Most of the patients had intermittent claudication, and there were patients with critical limb ischemia (CLI). Iida et al. ${ }^{20}$ reported that $43 \%$ of CLI patients had isolated BTK lesions. If the present study had included more CLI patients with isolated BTK lesions, the diagnostic performance of oscillometric ABI might have been lower. However, ABI would not be needed in CLI patients who have overt symptoms, such as ischemic color changes, ulcers or gangrene. The main use for

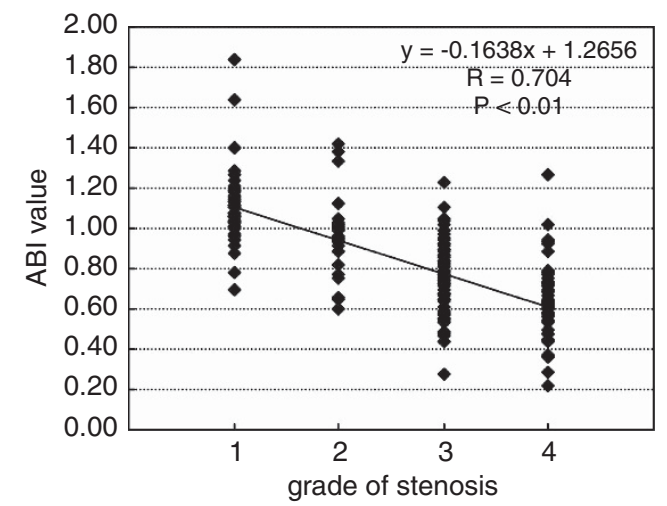

Figure 2 The relationship between the $A B I$ and severity of the arterial stenosis in the lower extremity. (grade $1,0-50 \%(N=45)$; grade 2, $50-75 \%(N=28)$; grade 3, 75-99\% $(N=70)$; grade $4,100 \%(N=64)) N$, number of limbs. A full color version of this figure is available at the Hypertension Research journal online. 
ABI could be screening of patients with intermittent claudication or asymptomatic arterial stenosis, and the present results showed the usefulness of $\mathrm{ABI}$ in these types of patients.

In conclusion, oscillometric ABI measured in the present study, which included a large number of PAD patients, offered high diagnostic ability for lower arterial stenosis. The diagnostic ability of ABI may be impaired by the presence of DM.

\section{CONFLICT OF INTEREST}

The authors declare no conflict of interest.

1 Ankle Brachial Index Collaboration, Fowkes FG, Murray GD, Butcher I, Heald CL, Lee RJ, Chambless LE, Folsom AR, Hirsch AT, Dramaix M, de Backer G, Wautrecht JC, Kornitzer M, Newman AB, Cushman M, Sutton-Tyrrell K, Fowkes FG, Lee AJ, Price JF, d'Agostino RB, Murabito JM, Norman PE, Jamrozik K, Curb JD, Masaki KH, Rodríguez BL, Dekker JM, Bouter LM, Heine RJ, Nijpels G, Stehouwer CD Ferrucci L, McDermott MM, Stoffers HE, Hooi JD, Knottnerus JA, Ogren $M$ Hedblad B, Witteman JC, Breteler MM, Hunink MG, Hofman A, Criqui MH, Langer RD, Fronek A, Hiatt WR, Hamman R, Resnick HE, Guralnik J, McDermott MM. Ankle brachial index combined with Framingham Risk Score to predict cardiovascular events and mortality: a meta-analysis. JAMA 2008; 300: 197-208.

2 Aboyans V, Criqui MH, Abraham P, Allison MA, Creager MA, Diehm C, Fowkes FG, Hiatt WR, Jönsson B, Lacroix P, Marin B, McDermott MM, Norgren L, Pande RL, Preux PM, Stoffers HE, Treat-Jacobson D. Measurement and interpretation of the ankle-brachial index: a scientific statement from the American Heart Association. Circulation 2012; 126: 2890-2909.

3 Mohler ER 3rd, Treat-Jacobson D, Reilly MP, Cunningham KE, Miani M, Criqui MH, Hiatt WR, Hirsch AT. Utility and barriers to performance of the ankle-brachial index in primary care practice. Vasc Med 2004; 9: 253-260.

4 Cortez-Cooper MY, Supak JA, Tanaka H. A new device for automatic measurements of arterial stiffness and ankle-brachial index. Am J Cardiol 2003; 91: 1519-1522.

5 Richart T, Kuznetsova T, Wizner B, Struijker-Boudier HA, Staessen JA. Validation of automated oscillometric versus manual measurement of the ankle-brachial index. Hypertens Res 2009; 32: 884-888.

6 Beckman JA, Higgins CO, Gerhard-Herman M. Automated oscillometric determination of the ankle-brachial index provides accuracy necessary for office practice. Hypertension 2006; 47: 35-38.
7 Kornø M, Eldrup N, Sillesen H. Comparison of ankle-ebrachial index measured by an automated oscillometric apparatus with that by standard doppler technique in vascular patients. Eur J Vasc Endovasc Surg 2009; 38: 610-615.

8 Clairotte C, Retout S, Potier L, Roussel R, Escoubet B. Automated anklebrachial pressure index measurement by clinical staff for peripheral arterial disease diagnosis in nondiabetic and diabetic patients. Diabetes Care 2009; 32. 1231-1236.

9 Okamoto K, Oka M, Maesato K, Ikee R, Mano T, Moriya H, Ohtake T, Kobayashi S. Peripheral arterial occlusive disease is more prevalent in patients with hemodialysis: comparison with the findings of multidetector-row computed tomography. Am J Kidney Dis 2006; 48: 269-276.

10 Catalano C, Fraioli F, Laghi A, Napoli A, Bezzi M, Pediconi F, Danti M, Nofroni I, Passariello R. Infrarenal aortic and lower-extremity arterial disease: diagnostic performance of multi-detector row CT angiography. Radiology 2004; 231: 555-563.

11 Brockmann C, Jochum S, Sadick M, Huck K, Ziegler P, Fink C, Schoenberg SO Diehl SJ, Dual-energy CT. angiography in peripheral arterial occlusive disease. Cardiovasc Intervent Radiol 2009; 32: 630-637.

12 Mundt KA, Chambless LE, Burnham CB, Heiss G. Measuring ankle systolic blood pressure: validation of the Dinamap 1846 SX. Angiology 1992; 43: 555-566.

13 Lee BY, Campbell JS, Berkowitz P. The correlation of ankle oscillometric blood pressures and segmental pulse volumes to Doppler systolic pressures in arterial occlusive disease. J Vasc Surg 1996; 23: 116-122.

14 Mehlsen J, Wiinberg N, Bruce C. Oscillometric blood pressure measurement: a simple method in screening for peripheral arterial disease. Clin Physiol Funct Imaging 2008; 28: 426-429.

15 Ramanathan A, Conaghan PJ, Jenkinson AD, Bishop CR. Comparison of ankle-brachial pressure index measurements using an automated oscillometric device with the standard doppler ultrasound technique. ANZ J Surg 2003; 73: 105-108.

16 Nukumizu Y, Matsushita M, Sakurai T, Kobayashi M, Nishikimi N, Komori K. Comparison of doppler and oscillometric ankle blood pressure measurement in patients with angiographically documented lower extremity arterial occlusive disease. Angiology 2007; 58: 303-308.

17 Guo X, Li J, Pang W, Zhao M, Luo Y, Sun Y, Hu D. Sensitivity and specificity of ankle-brachial index for detecting angiographic stenosis of peripheral arteries. Circ $J$ 2008; 72: 605-610.

18 McDermott MM, Liu K, Criqui MH, Ruth K, Goff D, Saad MF, Wu C, Homma S, Sharrett AR. Ankle brachial index and subclinical cardiac and carotid disease: the Multi-Ethnic Study of Atherosclerosis. Am J Epidemiol 2005; 162: 33-41.

19 Everhart JE, Pettitt DJ, Knowler WC, Rose FA, Bennett PH. Medial arterial calcification and its association with mortality and complications of diabetes. Diabetologia 1988; 31: 16-23.

20 lida O, Soga Y, Yamauchi Y, Hirano K, Kawasaki D, Yamaoka T, Takahara M, Uematsu M. Clinical efficacy of endovascular therapy for patients with critical limb ischemia attributable to pure isolated infrapopliteal lesions. J Vasc Surg 2013; 57 974-981. 